\title{
Smart Pervasive Healthcare-Assistance in Daily Life
}

\author{
Yuki Tamura, Masaaki Hashida, Shuji Sannomiya, Makoto Iwata \\ Dept. of Information Systems Engineering \\ Kochi University of Technology \\ Kochi, JAPAN \\ Email: $\{125089$ s, 125092f\}@gs.kochi-tech.ac.jp, \{sannomiya.shuji, iwata.makoto\}@kochi-tech.ac.jp
}

\begin{abstract}
The proliferation of lifestyle-related diseases is now a serious social issue in the world. To prevent those diseases, we propose a surrounding healthcare environment where people can improve their lifestyle naturally. The proposed healthcare environment senses information from surrounding sensors, and detects opportunities to build up the user's health associated with the sensed information, and finally assists users in being aware of the opportunities naturally on the pervasive computing environment. To stimulate the users into natural improvement of their lifestyles, this paper proposes schemes to advise the stepwise improvement of the undesired lifestyle and to provide the natural stimulation through user's daily life such as shopping, exercising, and so on. As a result, the users without strong awareness of their health can improve their health conditions continuously and comfortably and raise the awareness of their health gradually and naturally until they can manage their health by themselves.
\end{abstract}

Keywords-healthcare; context-aware; pervasive computing environment; ad-hoc wireless; gradual improvement of lifestyle

\section{INTRODUCTION}

Nowadays, the epidemic of lifestyle-related diseases is a serious social issue in the world. As is well-known, it is easier to prevent than to cure. To prevent them efficiently, it is important for potential patients to be strongly aware of their undesirable lifestyles until they develop a disease or their disease conditions become more severe. For this reason, people should objectively recognize their health condition and living habits. In addition, when they meet opportunities to improve their objectionable lifestyle, people had better to be aware of the opportunities, and then behave appropriately. However, it is difficult for people to do so continuously without strong awareness of their healthcare because it is uncomfortable to change their daily lifestyle and awareness.

Therefore, we are aiming to establish a good healthcare environment where people without strong awareness of their healthcare can improve their daily behaviors continuously and comfortably and they can raise up the awareness of their health gradually and naturally until they can manage their health by themselves. To realize such kind of environment, this paper proposes a surrounding healthcare environment in which the modern ubiquitous information and communication technologies (ICTs) are fully utilized. The final objective of the proposed healthcare environment is to provide pervasive, personalized and context-aware ICT services to everybody, in anytime, at anyplace, as if they were the surrounding audio sound or the ambient air. As for the first step of our project, the system proposed in this paper detects opportunities to improve user's undesirable living habits based on his health condition and then assists the user in being aware of the opportunities in his daily life and acting for their effective improvement. By continuously living under the proposed environment, the user will be often aware of his health and behave for his good health, so that the user could be getting healthier without any support of medical doctor or nurse.

This paper is organized as follows. Section 2 discusses the related works, and Section 3 explains the overview of the surrounding healthcare environment and its technological requirements in terms of ICTs. Based on the requirements, Section 4 introduces the basic system architecture composed of sensing, thinking and acting modules, and Section 5 discusses about necessary technologies. Section 6 explains prototype and evaluations for feasibility of the proposed system. Finally, Section 7 explains conclusion.

\section{RELATED WORKS}

Several healthcare systems have been ever proposed and developed to help a potential patient improve his daily lifestyle. One of the simplest healthcare systems is a web-based health advice system which is mainly provided for anonymous users via the Internet [1]. However, this kind of system usually generates general advices suitable for ordinary people, so that the user has to reconsider those advices based on his personal health condition, and if necessary, the user has to modify them.

In order to support personalized advices, the health check and prediction system has been developed [2]. This system generates personalized healthcare advices based on user's health conditions by introducing a kind of smart fuzzy inference engine. However, a user has to check or measure his health conditions by himself.

In contrast to the health check and prediction system, a context aware system for ubiquitous healthcare services in a smart home has been developed [3]. In this system, PDA gathers vital data from wearable and home network devices and it provides healthcare services only at home. The systems may be used only when the user intends to utilize the system and improve his lifestyle.

With the recent advancement of wireless communication technology, a wearable healthcare support system has been proposed in [4]. This system aims at improving a user's lifestyle by recognizing his current context in real time night and day. The system realizes the diet, exercise, and sleep 
condition management service in which a timely instruction is provided based on the user's current context. In this system, wearable sensors are used to detect the user's activities of daily living as a context, so that the system cannot provide appropriate advices based on user's health condition.

In order to solve these problems inherent in previous proposals, a smart healthcare system is required to operate as follows: It must work at anyplace and timely whenever the user can act to improve his lifestyle without doctor's support. It must recommend the suitable action to the user based on his personal health conditions and lifestyle. User's current health condition must be sensed automatically by some wearable sensors. Therefore, in our surrounding healthcare environment proposed in this paper, the surrounding or wearable sensors sense a user's context and health conditions and then the user's mobile device detects a timely context suitable for user's lifestyle improvement. Finally, the environment makes natural approaches to help the user be aware of his undesirable lifestyle.

\section{OVERVIEW}

Our surrounding healthcare environment proposed in this paper aids users without the awareness of their health to improve their lifestyle comfortably and continuously. Therefore, the proposed environment assists user in being aware of the opportunities in the pervasive computing environment. For example, if a user with lack of vitamins and minerals goes to a supermarket and walks close to a vegetable juice which contains vitamins and minerals, a display in front of the user plays a promotional video about the juice as shown in figure 1. The proposed environment repeats this kind of assistance in daily life and then user will be often aware of his health and behave for his good health. As a result, the user can raise the awareness of their health. After that, the proposed environment reduces frequency of the assistances, so that the user can manage his health autonomously. To realize this environment, we introduce three key ideas.

The first one is a context-aware scheme [5] to detect the healthcare context which represents an opportunity to improve user's undesired lifestyle. If the proposed environment becomes context-aware, the user can easily behave for his

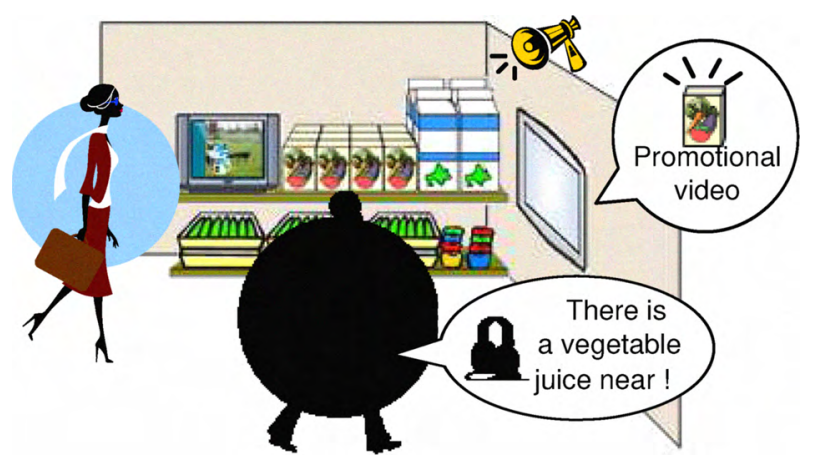

Fig. 1. An example daily situation and action in the surrounding healthcare environment. health improvement even if he is not usually keen on it. The healthcare context represents a set of personal health conditions and surrounding situations and is expressed in a simple type of language.

The second one is a natural assistance scheme under the pervasive computing environment to let the user be aware of the healthcare contexts and behave. Since it is uncomfortable for the user to often get advices only by user's mobile devices, the proposed environment assists user by some actions on surrounding computing devices such as advertising displays, electronic papers, loudspeakers. For example, the proposed environment assists a user by playing a promotional video bright on a display in front of the user, and then the juice bottle blinks its LED light. These are called acting devices in this paper. Moreover, to realize anytime-anywhere service, the acting devices must assist user in various situations of user's daily life even in the public area such as supermarket. In such place, the acting devices must assist several users at the same time with keeping satisfactions and health conditions of all users.

The final one is a stepwise assistance scheme for gradual improvement of lifestyle. Rapid improvement of lifestyles may be stressful for the user. For example, it is difficult for the user, who does not usually ingest vegetables, to ingest $400 \mathrm{~g}$ of vegetables per a day suddenly. Thus, we need to improve our lifestyle at each own suitable pace. At this point, the proposed healthcare environment must assist user manage his health by himself. The degree of the awareness about user's health is calculated based on the degree of excessive or lack (DEL). The degree of the awareness represents how much health awareness the user increases. The detail of the DEL is explained in the next section.

\section{ARCHITECTURE}

Because the proposed environment is a kind of contextaware system, the architecture of the surrounding healthcare environment can be designed by three kinds of subarchitectures as shown in figure 2 . At first, the sensing module gathers information about a user. By using the gathered information (we call it sensor data), the thinking module detects healthcare contexts proper for user's health condition and surrounding situations. After that, the acting module works to assist the user in being aware of the healthcare context and behaving appropriately.

\section{A. Sensing}

The sensing module gathers necessary information about a user from surrounding environments to detect the healthcare contexts properly. The sensing module is composed of wearable sensors and surrounding sensors. The wearable sensors collect user's vital data such as blood pulse, pressure, cardiac rate, and so on, while the surrounding sensors gather spatial situations around the user, e.g., healthcare-related objects close to the user, distance between the user and others, and so on. There are many research activities on intelligent sensing of human behavior and his environmental situation [6]. We 


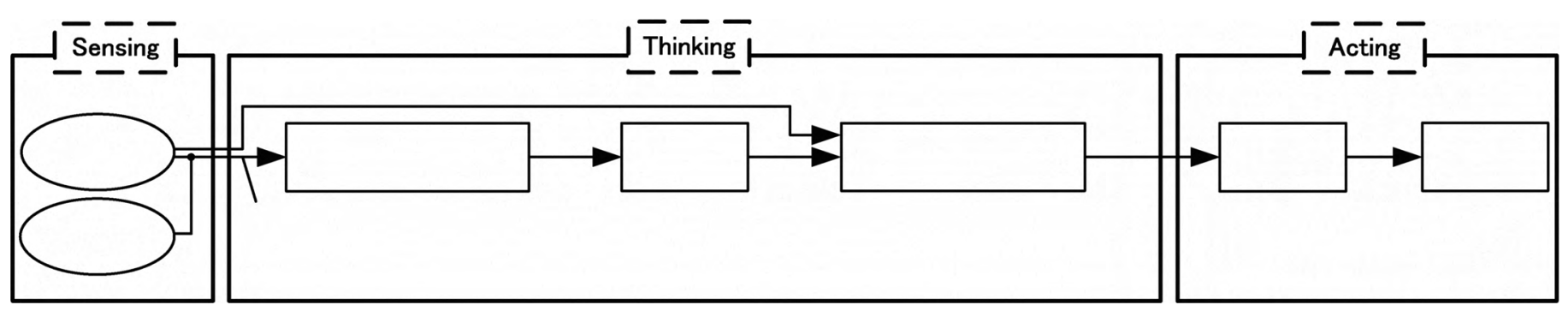

Fig. 2. Basic architecture of surrounding healthcare system.

can utilize those great progresses in our proposed system. However, since there is no completely intelligent sensor, our surrounding device allows user to input his preference directly.

\section{B. Thinking}

The thinking module detects the healthcare contexts based on sensor data. However, sensor data contain ambiguous data such as vital data. To deal with the data, the thinking module uses the simple distance-based fuzzy inference method proposed in [2].

At first, the personal context inference infers personal context including user's undesirable lifestyles and his preferences to detect healthcare contexts proper for him. The undesirable lifestyles include all nutrient intake, energy intake and energy consumption. Each lifestyle is associated with the DEL which is the amount of improvement. The DEL is defined as a value from $-100 \%$ to $+100 \%$. The $-100 \%$ means complete lacking, while $+100 \%$ means completely excessive intake or exercise. The DEL is calculated based on the distance between the actual intake or amount of exercise and the recommended value. The recommended value is decided based on a stepwise improvement scheme explained in Section Subsection V- $B$. For easy improvement of user's lifestyles, the preferences include food, sport, which activity user tends to do, which healthcare context user tends to be aware, and which assistance user tends to be aware. These can be acquired via direct user interface of the user device. However, he might not notice some of his own preferences. Thus the personal context inference partially infers his preferences as discussed in Subsection $\mathrm{V}-C$. Note that, a preference has a degree of like or hate (DLH) which is $0 \%$ to $+100 \%$. The $0 \%$ means the user completely hates it, the $+100 \%$ means the user completely likes it.

Next, the healthcare context detection traces surrounding situations based on environmental data and activity data and detects healthcare contexts. Here, multiplex states associated with contexts are analyzed by a finite state machine (FSM) as discussed in Subsection V- $D$.

\section{Acting}

The acting module assists some users by using acting devices so that they will be easily aware of the healthcare contexts.

To assist some users, the acting devices have some trigger information for detecting healthcare contexts and transfer to all users within the effective area. In the effective area, the acting device can act to the users, for example, who are standing within the line-of-sight distance for the display. After the acting devices gather healthcare contexts from them, the acting devices assist them for their good health.

Assistances are adaptively generated according to environments or situations. Therefore, acting devices coordinate each other and assist by the most effective combination of actions. In this paper, the combination of actions is called the scenario. The acting module plans the scenario proper for surrounding environment or situations on the scenario planner, and then acts according to the scenarios on the actor.

\section{UniQue HeAlThCARE Functions}

Based on the system architecture described in the previous section, several unique healthcare functions are introduced in our system. This section explains these major functions.

\section{A. Distance-Based Fuzzy Inference Method}

To deal with fuzzy data such as vital data, the personal context inference uses the simple distance-based fuzzy inference method. In this method, all fuzzy input data value are expressed by one of 5 fuzzy values, which are zero(Z), slight(S), slightly large(SL), large(L), and very large(VL). Fuzzy inference rules as shown in figure 3 are applied to the input fuzzy value, and the inference method calculates distance $D_{0}$ between an inference rule $x_{i j}$ and the input fuzzy value $x_{j}$ by the following formula. Note that the $x_{i j}$ is a fuzzy value of the $\mathrm{i}$-th rule of the $\mathrm{j}$-th item of a inference rule, and the $x_{j}$ is a set of input fuzzy values for the $\mathrm{j}$-th item.

$$
D_{0}=\left[\begin{array}{c}
d_{0} \\
d_{1} \\
\vdots \\
d_{n-1}
\end{array}\right], d_{i}=\sum_{j=0}^{m-1}\left|x_{i j}-x_{j}\right|
$$

After that, an inference result $y_{0}$ is calculated by the following formula.

$$
y_{0}=\frac{\sum_{i=0}^{n-1}\left[c_{i} \Pi_{j=0, j \neq i}^{n-1} d_{j}\right]}{\sum_{i=0}^{n-1}\left[\Pi_{j=0, j \neq i}^{n-1} d_{j}\right]}
$$

where $c_{i}$ denotes consequents of the inference, e.g. the value of the risk and the advice shown in figure 3 . The algorithm of the inference engine is very flexible because the simple 


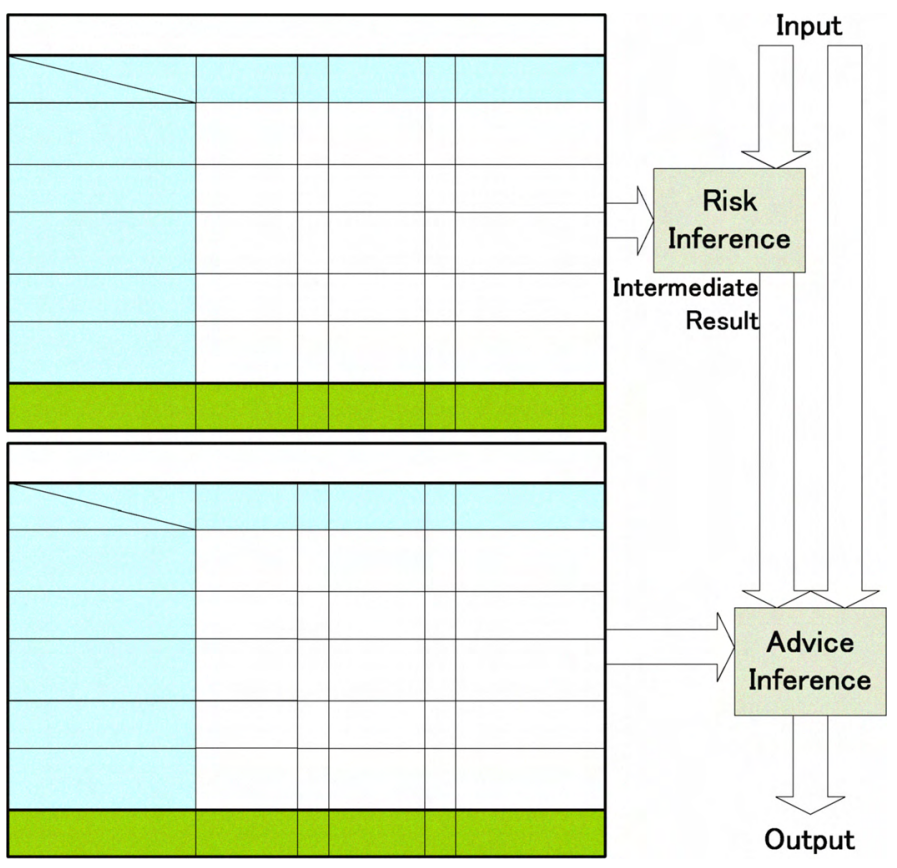

Fig. 3. Multistage inference engine using simple distance-based fuzzy inference method.

distance-based fuzzy inference turns an inference into another inference by using different inference rule.

In addition, we can use the inference result as an input data of other inferences, and then integrate several inference engines by piling each inference engine as shown in figure 3. Based on this multistage inference engine, if a part of an inference processing is the same as that of other inference processing on earlier stages, the same part can be skipped by reusing the result of the other earlier stages. Therefore, the total amount of the calculation on the inference can be reduced. Moreover, the reuse of the intermediate result makes it easy to embed a new inference in the inference engine.

Note that every variable such as DEL and DLH is defined as a normalized value from zero to one, because all functions in the thinking module are based on the simple distance-based fuzzy inference method. In our research, $-100 \%$ of the DEL is represented as 0 , while $+100 \%$ of the DEL is represented as $1.0 \%$ of the DLH is represented as 0 , while $+100 \%$ of the DLH is represented as 1 .

\section{B. Personal Context Inference Engine for Stepwise Improve- ment}

Rapid change of lifestyle may provide stresses to users and load to user's body. Therefore, the healthcare environment must assist him in improving his lifestyle gradually. At first, the system sets the amount of improvement which should be achieved for a fixed term (a recommended value) and assists user to behave to get the value as his habit. The improving style doesn't provide load to user's body. For example, if $400 \mathrm{~g}$ vegetables are too much for a user to eat, the inference engine

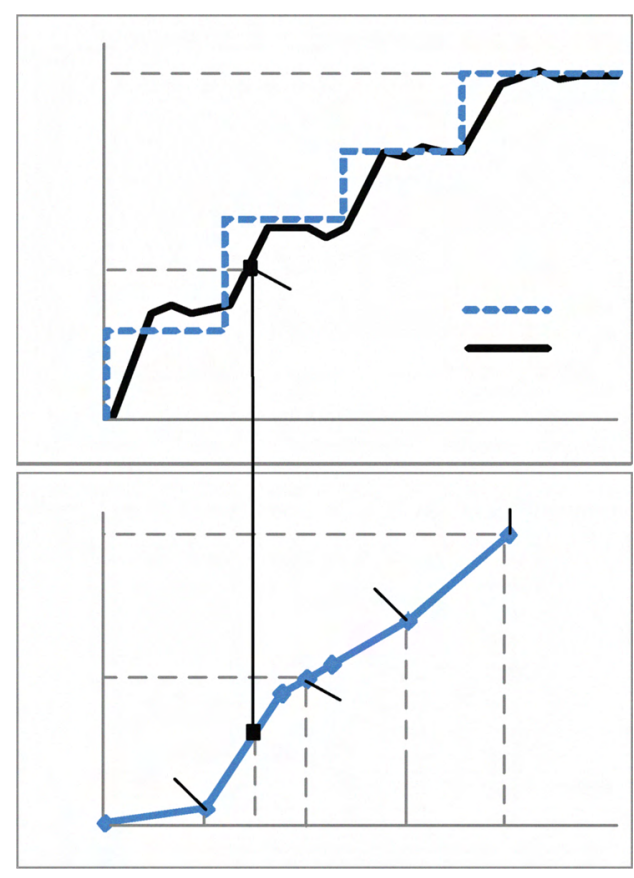

Fig. 4. Assistance for stepwise improvement.

sets $100 \mathrm{~g}$ a day as a recommended value. If $100 \mathrm{~g}$ is established as his habit or the fixed term is passed, the system sets next recommended value $R_{i}^{\prime}$ by formula 3 dealing with the time taken to establish the first recommended value. In the way, the improving style can provide no stresses to users. Note that $R_{i}$ is a value recommended by doctors or nurses, $A_{i-1}+\frac{\partial A_{i-1}}{\partial t}$ is a recommended value based on an actual improvement pace by the user, where $A_{i-1}$ is the actual value of the present lifestyle and $\frac{\partial A_{i-1}}{\partial t}$ is improvement rate for a fixed term until now, and $\alpha$ is a coefficient varying according to user's personal characteristics or situations.

$$
R_{i}^{\prime}=\frac{R_{i}+\alpha\left(A_{i-1}+\frac{\partial A_{i-1}}{\partial t}\right)}{1+\alpha}
$$

The upper graph of figure 4 shows the stepwise improvement of user's vegetable consumption. After that, the system evaluates the DEL. It is gotton by checking the curve such as shown the lower graph of figure 4 . The curve is given by the medical doctors or experts. For example, if a user should eat $300 \mathrm{~g}$ a day, that is recommended value, and eats only $250 \mathrm{~g}$ today, the DEL is $-35 \%$. Note that, the fuzzy data of the result is 0.35 . With this scheme, the users can improve their lifestyles comfortably without strong stress.

\section{Personal Context Inference Engine for Assistance based on Preference}

The engine infers user's preferences, so that the user can demand assistance without stresses. These preferences can be learned by frequency of user's activity. At first, a history of user's activities is accumulated. Next, the engine extracts the history data for a fixed term until now, and then calculates the frequency which represents percentage per user's activity. The 
$100 \%$ means that the user tends to do the activity, while the $0 \%$ means that the user doesn't like to do the activity. After that, a fuzzy inference rule is applied to the frequencies. The DLH of a preference is then calculated.

\section{Healthcare Context Detection}

In the healthcare context detection, user's surrounding contexts are analyzed in real time in order to extract healthcare context which expresses opportunities such as 'drinking vegetable juice' and 'taking stairs instead of elevator'. According to the user's behaviors, the multiple states of the user might change. For example, when a user enters a supermarket to buy something, the location of the user is analyzed as 'in the entrance of a supermarket' and the action of the user is analyzed as 'shopping'. In the healthcare context detection, such multiplex states are analyzed by using a finite state machine (FSM).

The FSM has states for contexts such as location and activities, and the states are transited according to the sensor data. The meaningful patterns of the multiple states are predesigned and associated with the healthcare contexts, and thus the healthcare contexts can be detected when the actual states of the user match with the predesigned FSM patterns. In this case, several healthcare contexts may be detected, and thus the most appropriate one should be selected. To quantify the satisfaction of the healthcare context, requirement degree $\left(D_{R}\right)$ is calculated with the following algorithm based on the amount of predicted intake or exercise $\left(A_{P}\right)$ and the amount of upper limit of the intake or exercise $\left(A_{L}\right)$, and the amount of satisfaction $\left(D_{S}\right)$. Note that one healthcare context may indicate several lifestyles such as intake and exercise, and $A_{P i}$, $A_{L i}$, and $D_{S i}$ denotes the amounts for each lifestyle $i$.

For all detected healthcare context, the following task is executed after $D_{R}$ is initialized to zero;

1) $A_{P i}$ is inferred;

2) If $A_{P i}$ is larger than $A_{L i}$;
a) $D_{S i}=0$
b) $D_{R}=0$
c) finish the task

3) Otherwise

a) $D_{S i}=\left(A_{P i} / A_{L i}\right) \times|D E L|$

b) $D_{R}+=D_{S i}$

After the above calculation, the detected healthcare context with the calculated $D_{R}$ are forwarded to the acting module.

\section{E. Scenario Planner}

In the scenario planner, actions and the sequence of the actions, which are collectively called scenario in this paper, are decided according to the detected healthcare context. The surrounding acting devices must act to all users within the same effective area where the users can be motivated by the devices. In addition, all acting devices must coordinate each other in order to assist all the users in public area naturally. To satisfy all the users, the most proper healthcare context is selected from the detected healthcare. Moreover, the acting

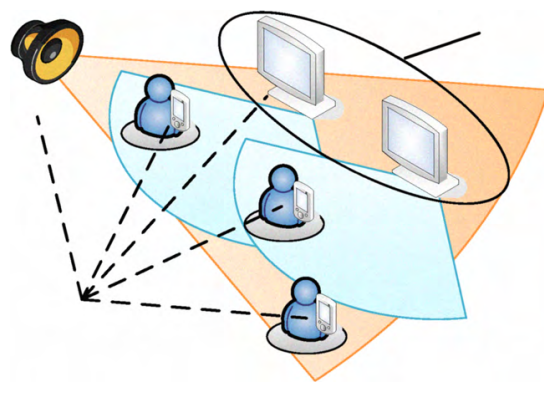

Fig. 5. An example group of acting devices.

devices within a given local area are layered according to size of their effective area, and the acting device with the biggest size within the area is selected as main device of the local area. The main devices communicate with each other and make the other local devices coordinate with each other. An example of this device layering is shown in figure 5 . This procedure is realized by the following algorithm.

1) The acting device collects the healthcare context when a user comes in its effective area and transfers the healthcare contexts to the main device.

2) The main device decides which users are assisted. If some of the users are in the intersection of two or more local areas, the following rules are applied.

a) The main device with the highest rank, or the biggest effective area take responsible for the assistance.

b) Other main devices notify the responsible device of the users and delete their users.

3) The $D_{R}$ of all users are summed up against each detected healthcare context.

4) One healthcare context with the largest sum of the $D_{R}$ is selected.

5) An adequate scenario is extracted from a database according to the selected healthcare context.

6) The responsible device asks itself and other devices to realize the scenario.

a) If the answer is 'no', the selected healthcare context is discarded. (Return to the step 4)

b) Otherwise, the responsible device details the scenario to be suitable for the preferences for the target users.

7) The responsible device issues the actions to the acting devices and the acting devices play the issued roll.

To realize such communication between acting devices including mobile devices, the mobile communication protocols such as mobile IPv6 and NEMO should be used. Such protocols can establish the communication within a given local area. These are the reason why the surrounding healthcare environment should be ad-hoc network.

\section{Prototype System}

We are now implementing a prototype system of our surrounding healthcare environment. Ideally, each sub- 


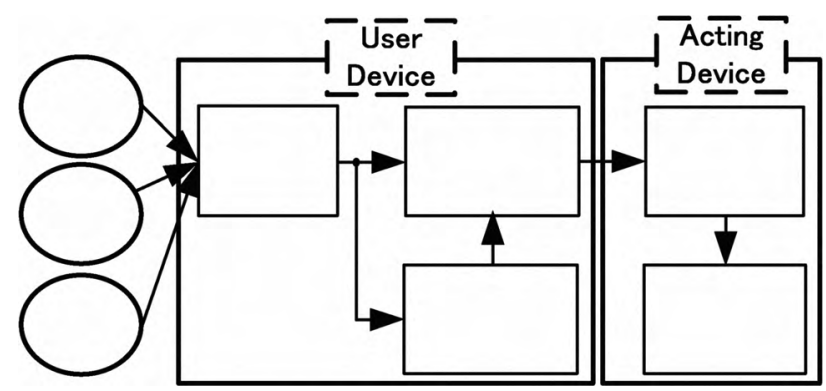

Fig. 6. The schematics of prototype system.

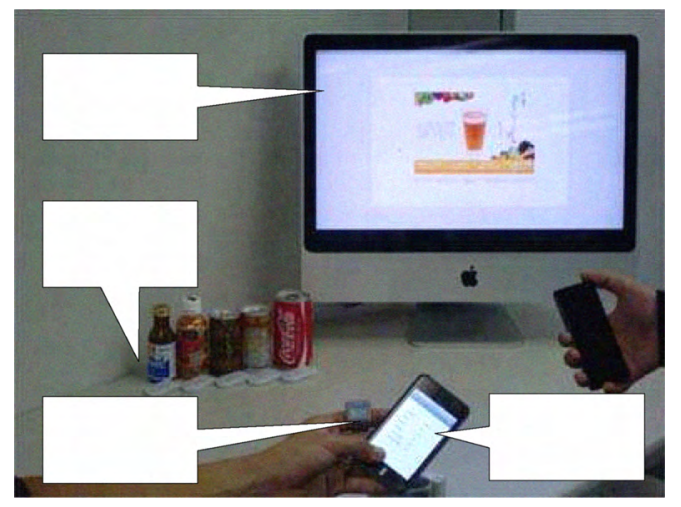

Fig. 7. Prototype of surrounding healthcare environment.

architecture shown in figure 2 should be realized on a single smart device communicating each other on a wireless ad-hoc network. However, the existing wireless devices and sensor nodes have some limited functions. Thus, our prototype system being built was modified as shown in figure 6 . In the figure, an additional filtering module is a kind of communication/user interface to compensate for primitive functions of RFID tags and vital sensors. This module generates available data for the healthcare context detection and the personal context inference based on the sensor data.

In the current prototype system, we adopt Apple iPod touch as a user device, which is equipped with Wi-Fi and Bluetooth communication interface. All necessary healthcare functions on the user device are implemented by ObjectiveC. By attaching an active RFID to iPod touch, each user's location is roughly identified by RFID readers located in the experimental space. Furthermore, each user has a wearable blood pulse meter and blood pressure meter, each of which is equipped with Wi-Fi to transfer measured vital data to the server. These wearable sensors and RFIDs work as the sensing module. A part of the thinking module is implemented on the user device. The acting module is implemented on the iMac, acting device. The user devices and the acting devices communicate on the ad-hoc network by Bluetooth and Wi-Fi. Figure 7 shows a photo of our prototype system.

By using the prototype, we verified the basic functionality of our experimental system. In the next step of evaluation, we are planning to setup our system in actual location such as supermarket or convenient store and to evaluate practical usability and effectiveness of health promotion in corporation with medical or healthcare doctors.

\section{CONCLUSION}

This paper proposed a framework of surrounding healthcare environment to support user's health promotion in daily life. By utilizing the proposed system, people without strong awareness of their health can improve their undesired lifestyles comfortably and continuously and raise the awareness of their health gradually and naturally until they can manage their health by themselves. To enable users to use our system in daily life, our healthcare system is basically realized on the pervasive computing environment based on the wireless adhoc network and introduces unique functions: the surrounding action scenario for the user's community and the smart assistance for stepwise improvement of user's lifestyle.

However our lifestyle is influenced by other close person's lifestyle because we often contact with other person such as family, friends, and boss. For example, children only have foods prepared by their mother. Thus, the surrounding environment should be able to assist user by dealing with this indirect assistance of personal community in the future.

To put our proposed framework to practical use, further investigation must be needed. Healthcare advices or actions generated by the system must be guaranteed to be safe in terms of medical point of view. It is also quite significant to prevent malicious invasion of private information such as personal health condition, especially on vulnerable wireless network. The more intelligent sensor devices must be developed to recognize events occurred around user's daily activities. In terms of establishing green computation and communication environment, ultra-low-power and dependable computing and networking technology[7] must be also investigated further.

\section{ACKNOWLEDGMENT}

This work was supported in part by Core Research for Evolutional Science and Technology (CREST), Japan Science and Technology Agency (JST).

\section{REFERENCES}

[1] "Electronic Preventive Services Selector (ePSS) for WEB : Search for Recommendations", http://epss.ahrq.gov/ePSS/GetResults.do?new= true, AHRQ, Feburary 2009

[2] X. Yu and S. Wang, "A Health Check and Prediction System for LifestyleRelated Disease Prevention,” icicic'06, pp.321-324, August 2006.

[3] D. Kang, K. Kang, H. Lee, E. Ko and J. Lee, "A Systematic Design Tool of Context Aware System for Ubiquitous Healthcare Service in a Smart Home," FGCN2007, pp.49-54, December 2007.

[4] K. Ouchi, T. Suzuki, A. Moriya and K. Kameyama, "Healthcare Services Using a Wealable Device," IPSJ SIG Technical Report-UBI, Vol.2007, No.14, pp.29-36, February 2007(in Japanese).

[5] S. Loke, "Context-Aware Pervasive Systems: Architectures for A New Breed of Applications," AUERBACH, December 2006.

[6] T. Choudhury, G. Borriello, S. Consolvo, D. Haehnel, B. Harrison, B. Hemingway, J. Hightower, P. P. Klasnja, K. Koscher, A. LaMarca, J. A. Landay, L. LeGrand, J. Lester, A. Rahimi, A. Rea, D. Wyatt, "The Mobile Sensing Platform: An Embedded Activity Recognition System," IEEE Pervasive Computing, vol.7, No.2, pp.32-41, June 2008.

[7] M. Iwata, H. Shima, and S. Sannomiya, "Self-timed Stream Processor for Surrounding Computing Environment," The 2007 International Conference on Parallel and Distributed Processing Techniques and Applications, pp.655-660, June 2007. 